\title{
Changes in performance of small bowel capsule endoscopy based on nationwide data from a Korean Capsule Endoscopy Registry
}

Su Hwan Kim ${ }^{1}$, Yun Jeong Lim², Junseok Park ${ }^{3}$, Ki-Nam Shim ${ }^{4}$, Dong-Hoon Yang 5 , Jaeyoung Chun ${ }^{6}$, Jin Su Kim7, Hyun Seok Lee ${ }^{8}$, Hoon Jai Chun ${ }^{9}$, and Research Group for Capsule Endoscopy/Small Bowel Endoscopy

Department of Internal Medicine, ${ }^{1}$ Seoul Metropolitan Government Seoul National University Boramae Medical Center, Seoul; ${ }^{2}$ Dongguk University Ilsan Hospital, Goyang; ${ }^{3}$ Soonchunhyang University College of Medicine, Seoul; ${ }^{4}$ Ewha Womans University School of Medicine, Seoul; ${ }^{5}$ University of Ulsan College of Medicine, Seoul; ${ }^{6}$ Yonsei University College of Medicine, Seoul; ${ }^{7}$ College of Medicine, The Catholic University of Korea, Seoul; ${ }^{8}$ School of Medicine, Kyungpook National University, Daegu; ${ }^{9}$ Korea University College of Medicine, Seoul, Korea

Received: September 18, 2019 Revised : November 18, 2019 Accepted: December 6, 2019

Correspondence to

Yun Jeong Lim, M.D.

Department of Internal

Medicine, Dongguk University

Ilsan Hospital, 27 Dongguk-ro,

Ilsandong-gu, Goyang 10326,

Korea

Tel: +82-31-961-7133

Fax: +82-31-961-7730

E-mail: drlimyj@gmail.com

https://orcid.org/0000-0002-

$3279-332 \mathrm{X}$
Background/Aims: Capsule endoscopy (CE) is widely used for the diagnosis of small bowel diseases. The clinical performance and complications of small bowel $\mathrm{CE}$, including completion rate, capsule retention rate, and indications, have been previously described in Korea. This study aimed at estimating the recent changes in clinical performance and complications of small bowel CE based on 17-year data from a Korean Capsule Endoscopy Registry.

Methods: CE registry data from 35 hospitals were retrospectively analyzed. Clinical information, including completion rate, capsule retention rate, and indications, was collected and analyzed. In addition, the most recent 5-year data for CE examinations were compared with the previous 12-year data.

Results: A total of 4,650 CE examinations were analyzed. The most common indication for CE was obscure gastrointestinal bleeding (OGIB). The overall incomplete examination rate was $16 \%$ and the capsule retention rate was 3\%. Crohn's disease was a risk factor for capsule retention. Inadequate bowel preparation was significantly associated with capsule retention and incomplete examination. An indication other than OGIB was a risk factor for incomplete examination. A recent increasing trend of CE diagnosis of Crohn's disease was observed. The most recent 5-year incomplete examination rate for CE examinations decreased compared with that of the previous 12 years.

Conclusions: The 17-year data suggested that CE is a useful and safe tool for diagnosing small bowel diseases. The incomplete examination rate of $\mathrm{CE}$ decreased with time, and OGIB was consistently the main indication for CE. Inadequate bowel preparation was significantly associated with capsule retention and incomplete examination.

Keywords: Capsule endoscopy; Intestine, small; Retention; Bowel preparation

\section{INTRODUCTION}

Since Capsule endoscopy (CE) was introduced in 2000, it has been widely used as the main tool for diagnosing small bowel diseases [1]. Although CE is useful for diag- nosing small bowel diseases, it has some limitations including incomplete examinations and capsule retention [2-4]. Occasionally, capsules cannot reach the cecum due to various causes, thus leading to incomplete examination of the small bowel. Another problem is capsule re- 
tention. Most patients in whom capsule retention occurs are asymptomatic; however, some require surgical or enteroscopic capsule removal [4-9]. The clinical performance and complications of small bowel CE include completion rate, capsule retention rate, and indications $[2,3]$; these have also been described in Korea [10]. This study aimed to investigate the clinical performance and complications of small bowel CE based on 17-year data from a Korean Capsule Endoscopy Registry. Furthermore, recent changes in the clinical performance and complications of small bowel CE were also evaluated.

\section{METHODS}

CE registry data from 35 hospitals, collected between October 2002 and April 2019, were retrospectively analyzed. Clinical information, including demographic data, completion rate, capsule retention rate, indications, CE diagnosis, and bowel preparation data, was collected and analyzed. From September 2014, health insurance benefits were provided to patients with obscure gastrointestinal bleeding (OGIB) in Korea. However, for patients with small bowel tumor(s), Crohn's disease, and other small bowel diseases, a patient burden of $80 \%$ was applied. As such, data from the most recent 5 years (September 2014 to April 2019) were compared with those from the previous 12 years (October 2002 to August 2014).

Indications for CE included OGIB, abdominal pain, chronic diarrhea, small bowel tumor(s), Crohn's disease, weight loss, protein-losing enteropathy, and screening. OGIB was defined as bleeding of unknown cause that persisted or recurred after initial upper gastrointestinal endoscopy and colonoscopy with negative results [11,12]. In addition to overt bleeding (melena or hematochezia), occult bleeding (persistent iron deficiency anemia or positive stool occult blood) was also considered OGIB $[12,13]$.

Before CE examinations, patients were offered a bowel preparation regimen according to clinician preferences. Options for the bowel preparation included nil per os (NPO), polyethylene glycol (PEG), PEG + ascorbic acid, sodium phosphate (NaP), picosulfate, or others. Bowel preparation quality was categorized by independent examiners as follows: excellent, $\geq 75 \%$ of the mucosa visualized; good, $50 \%$ to $74 \%$ of the mucosa visualized; fair,
$25 \%$ to $49 \%$ of the mucosa visualized; poor, $\leq 24 \%$ of the mucosa visualized [14]. For the purpose of study analysis, excellent or good bowel preparation were deemed adequate, and fair or poor preparation were deemed inadequate [15]. Comparison was performed between adequate and inadequate bowel preparation quality.

CE diagnoses were based on CE structured terminology. Complete examination was defined when the capsule reached the cecum during the recording time $[2,3]$. Capsule retention was defined when the capsule remained in the digestive tract for $>2$ weeks $[2,10]$. Completion and capsule retention rates were calculated, and risk factors affecting incomplete examination and capsule retention were also investigated. Acquisition of informed consent was exempted and the study design was approved by the Institutional Review Board of Seoul Metropolitan Government Seoul National University Boramae Medical Center (approval number: 20190802/10-2019-62/091).

Data are expressed as mean \pm standard deviation $(\mathrm{SD})$ for continuous variables and number (\%) for categorical variables. Risk factors for incomplete examination and capsule retention were analyzed using logistic regression models. Statistical analysis was performed using SPSS version 20.0 (IBM Corporation, Armonk, NY, USA).

\section{RESULTS}

\section{Demographics and indications}

A total of 4,650 CE examinations were analyzed. The mean \pm SD age of the study population was $53.5 \pm 18.8$ years, and $60.3 \%$ was male. The overall incomplete examination rate was $16.3 \%$, and the capsule retention rate was $3 \%$. The incomplete examination rate of recent CE examinations (from September 2014 to April 2019) decreased compared with that of previous examinations (9.4\% vs. $18.9 \%, p<0.001$ ) (Table 1). The most common indication for CE was OGIB (64.4\%). Patients in whom the indication for CE was OGIB accounted for $68.8 \%$ in recent $\mathrm{CE}$ examinations and $62.7 \%$ in previous $\mathrm{CE}$ examinations $(p<0.001)$ (Table 2$)$.

\section{CE diagnoses}

Positive CE diagnosis was obtained in 63.8\% CE examinations, while negative (normal) CE diagnosis was ob- 
Table 1. Demographic information and incomplete examination and retention rates

\begin{tabular}{lcrr}
\hline Variable & \multicolumn{1}{c}{ Total } & Aug. 31, 2014 & Sep. 1, 2014 \\
\hline Age & $53.5 \pm 18.8$ & $53.1 \pm 18.1$ & $55.0 \pm 20.5$ \\
Male sex & $2,804(60.3)$ & $2,067(61.1)$ & $681(58.2)$ \\
Incompletion & $678(16.3)$ & $570(18.9)$ & $108(9.4)^{\mathrm{a}}$ \\
Retention & $125(3.0)$ & $105(3.2)$ & $20(2.6)$ \\
\hline
\end{tabular}

Values are presented as mean \pm SD or number (\%).

${ }^{\mathrm{a}}$ Decreased trend.

Table 2. Indications for capsule endoscopy

\begin{tabular}{|c|c|c|c|}
\hline Variable & Total & Aug. 31, 2014 & Sep. 1, 2014 \\
\hline Obscure GI bleeding & $2,965(64.4)$ & $2,110(62.7)$ & $790(68.8)^{\mathrm{a}}$ \\
\hline Overt & $2,445(53.1)$ & $1,755(52.2)$ & $638(55.6)$ \\
\hline Occult & $520(11.3)$ & $355(10.5)$ & $152(13.2)$ \\
\hline Abdominal pain & $724(15 \cdot 7)$ & $594(17.7)$ & $117(10.2)$ \\
\hline Crohn's disease & $212(4.6)$ & $119(3.5)$ & $86(7.5)^{\mathrm{a}}$ \\
\hline Small bowel tumor & $166(3.6)$ & $122(3.6)$ & $42(3.7)$ \\
\hline Chronic diarrhea & $171(3.7)$ & $118(3.5)$ & $51(4 \cdot 4)$ \\
\hline Weight loss & $19(0.4)$ & $18(0.5)$ & $1(0.1)$ \\
\hline Cancer of unknown primary site & $4(0.1)$ & $3(0.1)$ & $\mathrm{O}$ \\
\hline Protein-losing enteropathy & $15(0.3)$ & $6(0.2)$ & $7(0.6)$ \\
\hline Behcet's disease, TB enteritis & $44(1.0)$ & $21(0.6)$ & $23(2.0)$ \\
\hline Ileus & $13(0.3)$ & $5(0.1)$ & $8(0.7)$ \\
\hline Screening & $214(4.6)$ & $209(6.2)$ & $2(0.2)$ \\
\hline
\end{tabular}

Values are presented as number (\%).

GI, gastrointestinal; TB, tuberculous.

${ }^{\mathrm{a}}$ Increased trend.

tained in 36.2\% CE examinations. Recent CE examinations yielded normal results in $28.4 \%$, which was lower than the normal results in previous CE examinations $(38.5 \%)(p<0.001)$. CE diagnoses are summarized in Table 3. A recent increasing trend of CE diagnosis of Crohn's disease was observed (6.3\% vs. $8.5 \%, p=0.014$ ). Regarding nonsteroidal anti-inflammatory drug (NSAID) enteropathy, significant changes with time were not observed (5.7\% vs. $6.5 \%, p=0.305)$.

\section{Factors associated with incomplete examination and capsule retention}

Factors associated with incomplete examination includ- ed inadequate bowel preparation and an indication other than OGIB (Table 4). Crohn's disease was a risk factor for capsule retention, and inadequate bowel preparation was significantly associated with capsule retention (Table 5). The capsule retention rate was the highest among patients with Crohn's disease (Table 6).

\section{Bowel preparation}

The quality of bowel preparation significantly differed according to bowel preparation regimen (Table 7). When the proportion of adequate bowel preparation after ingesting the bowel preparation regimen was compared pairwise with that after NPO, significant differences 
Table 3. Capsule endoscopic diagnosis

\begin{tabular}{|c|c|c|c|}
\hline Variable & Total & Aug. 31, 2014 & Sep. 1, 2014 \\
\hline Normal & $1,647(36.2)$ & $1,285(38.5)$ & $322(28.4)^{\mathrm{a}}$ \\
\hline \multicolumn{4}{|l|}{ Vascular lesions } \\
\hline Angiodysplasia, telangiectasia, Dieulafoy's lesion & $442(9 \cdot 7)$ & $349(10.4)$ & $87(7 \cdot 7)$ \\
\hline Bleeding of unidentified origin & $234(5.1)$ & $122(3.7)$ & $109(9.6)$ \\
\hline Varices & $12(0.3)$ & $10(0.3)$ & $2(0.2)$ \\
\hline \multicolumn{4}{|l|}{ Inflammatory lesions } \\
\hline Erosion, not signified & $365(8.0)$ & $233(7)$ & $130(11.5)$ \\
\hline Ulcer, not signified & $469(10.3)$ & $352(10.5)$ & $112(9.9)$ \\
\hline Hemorrhagic enteropathy & $31(0.7)$ & $28(0.8)$ & $3(0.3)$ \\
\hline Congestive enteropathy & $13(0.3)$ & $13(0.4)$ & $6(0.5)$ \\
\hline H-S purpura, vasculitis & $11(0.2)$ & $10(0.3)$ & $1(0.1)$ \\
\hline Ischemic enteritis & $9(0.2)$ & $6(0.2)$ & $3(0.3)$ \\
\hline NSAID enteropathy & $267(5.9)$ & $190(5.7)$ & $74(6.5)$ \\
\hline TB enteritis & $30(0.7)$ & $16(0.5)$ & $12(1.1)$ \\
\hline Behcet's enteritis & $33(0.7)$ & $22(0.7)$ & $11(1.0)$ \\
\hline Radiation enteritis & $9(0.2)$ & $8(0.2)$ & 0 \\
\hline Eosinophilic enteritis & $18(0.4)$ & $10(0.3)$ & $6(0.5)$ \\
\hline Crohn's disease & $316(6.9)$ & $211(6.3)$ & $96(8.5)^{b}$ \\
\hline CMUSE & $3(0.1)$ & $1(0)$ & $2(0.2)$ \\
\hline \multicolumn{4}{|l|}{ Tumor lesions } \\
\hline Lymphoid hyperplasia & $25(0.5)$ & $22(0.7)$ & $3(0.3)$ \\
\hline Lymphangiectasia & $30(0.7)$ & $17(0.5)$ & $12(1.1)$ \\
\hline Malignant tumor & $50(1.1)$ & $40(1.2)$ & $9(0.8)$ \\
\hline Submucosal tumor & $146(3.2)$ & $120(3.6)$ & $24(2.1)$ \\
\hline Polyp, adenomatous & $25(0.5)$ & $21(0.6)$ & $4(0.4)$ \\
\hline Polyp, non-neoplastic & $134(2.9)$ & $105(3.1)$ & $25(2.2)$ \\
\hline Peutz-Jeghers syndrome & $10(0.2)$ & $7(0.2)$ & $3(0.3)$ \\
\hline Hemangioma & $4(0.1)$ & $2(0.1)$ & $2(0.2)$ \\
\hline Lymphangioma & $3(0.1)$ & $2(0.1)$ & $1(0.1)$ \\
\hline Others & $220(4.8)$ & $138(4.1)$ & $82(7.2)$ \\
\hline
\end{tabular}

Values are presented as number (\%).

H-S, Henoch-Schönlein; NSAID, nonsteroidal anti-inflammatory drug; TB, tuberculous; CMUSE, cryptogenic multifocal ulcerous stenosing enteritis.

${ }^{\mathrm{a}}$ Decreased trend.

${ }^{\mathrm{b}}$ Increased trend.

were identified for PEG 4 L, PEG + ascorbic acid $2 \mathrm{~L}$, and $\mathrm{NaP}(p=0.017, p<0.001, p=0.028$, respectively). When
Bonferroni correction was applied for multiple comparisons $(p<0.0125)$, the proportion of adequate bowel 
Table 4. Factors associated with incomplete examination

\begin{tabular}{|c|c|c|c|c|c|c|}
\hline \multirow{2}{*}{ Variable } & \multicolumn{3}{|c|}{ Crude } & \multicolumn{3}{|c|}{ Age and sex adjusted } \\
\hline & OR & $95 \%$ CI & $p$ value & OR & $95 \% \mathrm{CI}$ & $p$ value \\
\hline Age & 1.005 & $1.001-1.009$ & 0.026 & & & \\
\hline Male sex & 0.911 & $0.771-1.076$ & 0.274 & & & \\
\hline \multicolumn{7}{|l|}{ Indication for capsule endoscopy } \\
\hline OGIB vs. others & 0.761 & $0.642-0.902$ & 0.002 & 0.712 & $0.597-0.850$ & $<0.001$ \\
\hline Crohn's disease vs. others & 0.864 & $0.574-1.301$ & 0.484 & 0.966 & $0.636-1.467$ & 0.871 \\
\hline Small bowel tumor vs. others & 0.827 & $0.523-1.308$ & 0.418 & 0.868 & $0.548-1.375$ & 0.548 \\
\hline \multicolumn{7}{|l|}{ Bowel preparation } \\
\hline Inadequate vs. adequate & 1.757 & $1.479-2.086$ & $<0.001$ & 1.750 & $1.472-2.080$ & $<0.001$ \\
\hline
\end{tabular}

OR, odds ratio; CI, confidence interval; OGIB, obscure gastrointestinal bleeding.

Table 5. Factors associated with capsule retention

\begin{tabular}{|c|c|c|c|c|c|c|}
\hline \multirow{2}{*}{ Variable } & \multicolumn{3}{|c|}{ Crude } & \multicolumn{3}{|c|}{ Age and sex adjusted } \\
\hline & OR & $95 \% \mathrm{CI}$ & $p$ value & OR & $95 \% \mathrm{CI}$ & $p$ value \\
\hline Age & 0.999 & $0.989-1.008$ & 0.814 & & & \\
\hline Male sex & 0.83 & $0.580-1.188$ & 0.309 & & & \\
\hline \multicolumn{7}{|l|}{ Indication for capsule endoscopy } \\
\hline OGIB vs. others & 1.083 & $0.745^{-1.575}$ & 0.677 & 1.092 & $0.743-1.604$ & 0.654 \\
\hline Crohn's disease vs. others & 2.264 & $1.226-4.182$ & 0.009 & 2.380 & $1.258-4.505$ & 0.008 \\
\hline Small bowel tumor vs. others & 0.213 & $0.030-1.533$ & 0.124 & 0.209 & $0.029-1.506$ & 0.120 \\
\hline \multicolumn{7}{|l|}{ Bowel preparation } \\
\hline Inadequate vs. adequate & 2.434 & $1.677-3.533$ & $<0.001$ & 2.412 & $1.657-3.510$ & $<0.001$ \\
\hline
\end{tabular}

OR, odds ratio; CI, confidence interval; OGIB, obscure gastrointestinal bleeding.

Table 6. Capsule retention rates according to indications for capsule endoscopy

\begin{tabular}{lc}
\hline Indications of capsule endoscopy & No. (\%) \\
\hline Obscure GI bleeding & $82(3.1)$ \\
Abdominal pain & $20(3.0)$ \\
Crohn's disease & $12(6.3)$ \\
Small bowel tumor & $1(0.7)$ \\
Screening & 0 \\
Chronic diarrhea & $2(1.3)$ \\
\hline
\end{tabular}

GI, gastrointestinal.

preparation was different only between PEG + ascorbic acid $2 \mathrm{~L}$ and NPO. PEG $2 \mathrm{~L}$ was previously the most commonly used regimen (47.9\%); however, recently, the PEG + ascorbic acid regimen has become the most popular (58.1\%) (Table 8).

\section{DISCUSSION}

In this registry-based study including 4,650 CE examinations, the incomplete examination and capsule retention rates were determined. This study revealed an incomplete examination rate of $16 \%$, which is similar to that reported in previous studies [2,3]. The capsule retention rate was $3 \%$, which is slightly higher than that reported in previous studies $[2,3]$. The incomplete examination rate of recent CE (September 2014 to April 2019) decreased compared with that of previous CE (October 2002 to August 2014). With the extended battery life of newer CE technologies, more capsules have reached the ileocecal valve and cecum, leading to a decrease in the number of incomplete examinations [16,17]. With technical advances in radiological examinations, such as computed tomography enterography and magnet- 
Table 7. Bowel preparation quality according to bowel preparation method

\begin{tabular}{|c|c|c|c|c|c|c|}
\hline Variable & NPO & $\mathrm{PEG}_{4} \mathrm{~L}$ & PEG $2 \mathrm{~L}$ & PEG + Asc $2 \mathrm{~L}$ & $\mathrm{NaP}$ & $p$ value \\
\hline Quality & & & & & & $<0.001$ \\
\hline Excellent & $149(21.4)$ & $104(16.1)$ & $165(10.8)$ & $92(13.5)$ & $89(30.3)$ & \\
\hline Good & $340(48.9)$ & $388(60.1)$ & $874(57.5)$ & $289(42.6)$ & $138(46.9)$ & \\
\hline Fair & $161(23.2)$ & $119(18.4)$ & $368(24.2)$ & $215(31.7)$ & $53(18.0)$ & \\
\hline Poor & $45(6.5)$ & $35(5.4)$ & $114(7.5)$ & $83(12.2)$ & $14(4.8)$ & \\
\hline Acceptability & & & & & & $<0.001$ \\
\hline Adequate & $489(70.4)$ & $492^{\mathrm{a}}(76.2)$ & $1,039(68.3)$ & $381^{\mathrm{a}}(56.1)$ & $227^{\mathrm{a}}(77.2)$ & \\
\hline Inadequate & $206(29.6)$ & $154(23.8)$ & $482(31.7)$ & $298(43.9)$ & $67(22.8)$ & \\
\hline$p$ value vs. NPO & Reference & 0.017 & 0.333 & $<0.001$ & 0.028 & \\
\hline
\end{tabular}

Values are presented as number (\%).

NPO, nil per os; PEG, polyethylene glycol; Asc, ascorbic acid; NaP, sodium phosphate.

${ }^{a}$ Statistically significant in comparison with NPO $(p<0.05)$.

Table 8. Trends in bowel preparation methods

\begin{tabular}{lccc}
\hline Variable & Total & Aug. 31, 2014 & Sep. 1, 2014 \\
\hline NPO & $704(17.5)$ & $560(19.2)$ & $144(12.9)$ \\
PEG 4 L & $658(16.3)$ & $546(18.7)$ & $111(10.0)$ \\
PEG 2 L & $1,553(38.5)$ & $1,398(47.9)$ & $155(13.9)$ \\
PEG + Asc 2 L & $681(16.9)$ & $34(1.2)$ & $646(58.1)$ \\
NaP & $297(7.4)$ & $297(10.2)$ & 0 \\
Picosulfate & $64(1.6)$ & $8(0.3)$ & $56(4.8)$ \\
Others & $77(1.9)$ & $77(2.7)$ & 0 \\
\hline
\end{tabular}

Values are presented as number (\%).

NPO, nil per os; PEG, polyethylene glycol; Asc, ascorbic acid; NaP, sodium phosphate.

ic resonance enterography, more patients with small bowel stricture(s) can be diagnosed before undergoing CE. However, the decrease in capsule retention rate over time was not statistically significant in our study. Moreover, our data lacked radiological examination results and, thus, analysis in this regard was not possible. The most common indication for CE was OGIB (64.4\%), which is consistent with results reported in previous studies $[2,3,10]$. In our study, recent CE examinations had more OGIB indications than previous CE examinations ( $p<0.001$ ), which can be explained by the inception of health insurance coverage for patients with OGIB in September 2014.

Recently, the incidence of Crohn's disease has been found to be increasing in Korea [18]. Our study revealed a recent increasing tendency of indications and CE diagnosis of Crohn's disease. CE diagnosis of NSAID enteropathy demonstrated an increasing tendency without statistical significance. The increase in NSAID enteropathy can be attributed to the aging population. In our study, the proportion of normal results was smaller in recent CE examinations than in previous examinations. Recent advances in CE technology with improved resolution may have led to more lesion detection and fewer patients diagnosed with normal findings.

Our study revealed that factors associated with incomplete examination were inadequate bowel preparation and indications other than OGIB, which is consistent 
with the results of a previous study [10]. With adequate bowel preparation, CE is more likely to be completed. Blood materials in patients with gastrointestinal bleeding appear to act as laxatives, thus helping the capsule proceed through the ileocecal valve. In our study, inadequate bowel preparation was significantly associated with capsule retention, which is also consistent with the results of previous studies $[2,10]$. The presence of stenotic portions in the small bowel may lead to both inadequate bowel preparation and capsule retention. Additionally, fecal materials stuck in the stenotic portion may result in capsule retention. Although causality was difficult to determine, our study revealed that inadequate bowel preparation was significantly associated with capsule retention. Crohn's disease was a risk factor for capsule retention and the capsule retention rate was the highest among patients with the indication of Crohn's disease. Special caution or using a patency capsule will be needed in patients with Crohn's disease.

In this study, bowel preparation quality was affected by the bowel preparation regimen. PEG $4 \mathrm{~L}$ or NaP more favorably affected bowel preparation than NPO; however, PEG + ascorbic acid resulted in worse bowel preparation than NPO. In our study, the recent data revealed that PEG + ascorbic acid regimen was the most widely used. This can be explained by the fact that recent guidelines recommend that $\mathrm{CE}$ should be performed as soon as possible (generally after colonoscopy) in patients with overt, obscure bleeding [11], and PEG + ascorbic acid has recently become the most popular regimen for colonoscopy in Korea [19]. The time interval from the ingestion of the bowel preparation regimen to performing CE can be delayed because performing colonoscopy and clinician's decision-making to perform CE after colonoscopy can take time. This time delay may adversely affect bowel preparation quality $[20,21]$, because debris and intestinal fluid can affect preparation of the distal small bowel [22].

Our study had some limitations, the first of which was its retrospective design and, as such, may have been susceptible to selection bias. Moreover, our data may have been susceptible to the under-reporting of complications. Second, our investigation was a multi-center, registry-based study, and the data collection methods used at each institution were not monitored. There was no auditing system to control data collection. However, this was a large study based on 17-year registry data.
Time trends were also investigated between recent and previous data.

In conclusion, our 17-year data suggest that CE is a useful and safe tool for diagnosing small bowel diseases. The incomplete examination rate of CE decreased with time and OGIB was consistently the primary indication for CE. Inadequate bowel preparation was significantly associated with capsule retention and incomplete examination.

\section{KEY MESSAGE}

1. The incomplete examination rate of capsule endoscopy decreased over time.

2. Obscure gastrointestinal bleeding was consistently the primary indication for capsule endoscopy.

3. Inadequate bowel preparation was significantly associated with capsule retention and incomplete examination.

\section{Conflict of interest}

No potential conflict of interest relevant to this article was reported.

\section{Acknowledgments}

This research was supported by a grant of the Korean Health Technology R \& D project through the Korean Health Industry Development Institute (KHIDI), funded by the Ministry of Health \& Welfare, Republic of Korea (grant number: HI19Co665).

\section{REFERENCES}

1. Iddan G, Meron G, Glukhovsky A, Swain P. Wireless capsule endoscopy. Nature 2000;405:417.

2. Liao Z, Gao R, Xu C, Li ZS. Indications and detection, completion, and retention rates of small-bowel capsule endoscopy: a systematic review. Gastrointest Endosc 2010;71:280-286.

3. Rondonotti E, Herrerias JM, Pennazio M, Caunedo A, Mascarenhas-Saraiva M, de Franchis R. Complications, limitations, and failures of capsule endoscopy: a review of 733 cases. Gastrointest Endosc 2005;62:712-716. 
4. Hoog CM, Bark LA, Arkani J, Gorsetman J, Brostrom O, Sjoqvist U. Capsule retentions and incomplete capsule endoscopy examinations: an analysis of 2300 examinations. Gastroenterol Res Pract 2012;2012:518718.

5. Rondonotti E, Spada C, Adler S, et al. Small-bowel capsule endoscopy and device-assisted enteroscopy for diagnosis and treatment of small-bowel disorders: European Society of Gastrointestinal Endoscopy (ESGE) Technical Review. Endoscopy 2018;50:423-446.

6. Li F, Gurudu SR, De Petris G, et al. Retention of the capsule endoscope: a single-center experience of 1000 capsule endoscopy procedures. Gastrointest Endosc 2008;68:174-18o.

7. Mitsui K, Fujimori S, Tanaka S, et al. Retrieval of retained capsule endoscopy at small bowel stricture by double-balloon endoscopy significantly decreases surgical treatment. J Clin Gastroenterol 2016;50:141-146.

8. Makipour K, Modiri AN, Ehrlich A, et al. Double balloon enteroscopy: effective and minimally invasive method for removal of retained video capsules. Dig Endosc 2014;26:646-649.

9. Van Weyenberg SJ, Van Turenhout ST, Bouma G, et al. Double-balloon endoscopy as the primary method for small-bowel video capsule endoscope retrieval. Gastrointest Endosc 2010;71:535-541.

10. Lim YJ, Lee OY, Jeen YT, et al. Indications for detection, completion, and retention rates of small bowel capsule endoscopy based on the 10-year data from the Korean Capsule Endoscopy Registry. Clin Endosc 2015;48:399-404.

11. Enns RA, Hookey L, Armstrong D, et al. Clinical practice guidelines for the use of video capsule endoscopy. Gastroenterology 2017;152:497-514.

12. Koulaouzidis A, Rondonotti E, Giannakou A, Plevris JN. Diagnostic yield of small-bowel capsule endoscopy in patients with iron-deficiency anemia: a systematic review. Gastrointest Endosc 2012;76:983-992.

13. Raju GS, Gerson L, Das A, Lewis B; American Gastroenterological Association. American Gastroenterological
Association (AGA) Institute technical review on obscure gastrointestinal bleeding. Gastroenterology 2007;133:16971717 .

14. Lapalus MG, Ben Soussan E, Saurin JC, et al. Capsule endoscopy and bowel preparation with oral sodium phosphate: a prospective randomized controlled trial. Gastrointest Endosc 2008;67:1091-1096.

15. Rosa BJ, Barbosa M, Magalhaes J, Rebelo A, Moreira MJ, Cotter J. Oral purgative and simethicone before small bowel capsule endoscopy. World J Gastrointest Endosc 2013;5:67-73.

16. Fisher LR, Hasler WL. New vision in video capsule endoscopy: current status and future directions. Nat Rev Gastroenterol Hepatol 2012;9:392-405.

17. Kwack WG, Lim YJ. Current status and research into overcoming limitations of capsule endoscopy. Clin Endosc 2016;49:8-15.

18. Park SH, Kim YJ, Rhee $\mathrm{KH}$, et al. A 30-year trend analysis in the epidemiology of inflammatory bowel disease in the Songpa-Kangdong District of Seoul, Korea in 19862015. J Crohns Colitis 2019;13:1410-1417.

19. Seong JH, Yoo JS, Lee KJ, et al. Formulation and management of poor bowel preparation: a survey study. Korean J Gastroenterol 2016;68:70-76.

20. Seo EH, Kim TO, Park MJ, et al. Optimal preparation-to-colonoscopy interval in split-dose PEG bowel preparation determines satisfactory bowel preparation quality: an observational prospective study. Gastrointest Endosc 2012;75:583-590.

21. Siddiqui AA, Yang K, Spechler SJ, et al. Duration of the interval between the completion of bowel preparation and the start of colonoscopy predicts bowel-preparation quality. Gastrointest Endosc 2009;69:700-706.

22. Adler SN, Farkash S, Sompolinsky Y, Gafanovich I, Goldin E, Bar-Gil Shitrit A. A novel purgative protocol for capsule endoscopy of the small bowel produces better quality of visibility than 21 of PEG: timing is of the essence. United European Gastroenterol J 2017;5:485-490. 